\title{
Photon-assisted Fano Resonance and Corresponding Shot-Noise in a Quantum Dot
}

\author{
Zhongshui Ma, ${ }^{1,2} \mathrm{Yu} \mathrm{Zhu},{ }^{1}$ Xin-Qi Li ${ }^{3}$ Tsung-han Lin, ${ }^{1}$ and Zhao-Bin $\mathrm{Su}^{4}$ \\ ${ }^{1}$ State Key Laboratory for Mesoscopic Physics and Department of Physics, Peking University, Beijing 100871, China \\ ${ }^{2}$ Advanced Research Cneter, Zhongshan University, Guangzhou 510275, China \\ ${ }^{3}$ NLSM, Institute of Semiconductors, CAS, Beijing 100083, China \\ ${ }^{4}$ Institute of Theoretical Physics, Chinese Academy of Science, Beijing 100080,China
}

\begin{abstract}
We have studied the Fano resonance in photon-assisted transport in a quantum dot and calculated both the coherent current and spectral density of shot noise. It is predicted, for the first time, that the shape of Fano profile will also appear in satellite peaks. It is found that the variations of Fano profiles with the strengths of nonresonant transmissions are not synchronous in absorption and emission sidebands. The effect of interference on photon-assisted pumped current has been also investigated. We further predict the current and spectral density of shot noise as a function of the phase, which exhibits an intrinsic property of resonant and nonresonant channels in the structures.
\end{abstract}

PACS numbers: 73.23.-b, 73.40.Gk

The phenomena of photon-assisted tunneling (PAT) have been intensively studied in a variety of mesoscopic systems. The main feature of PAT is that the monochromatic radiation induces additional tunneling processes when electrons exchange energy by absorbing or emitting photons, and the conductance displays a strong nonlinearity and develops sidebands 1, 2]. However, the previous studies of PAT have not considered the possibility of symmetric RT being violated in structures where the nonresonant transmission is taken part in the tunnelings between two reservoirs. Recently, Göres et al. 3 ] and Zacharia et al. [4] demonstrated in their experiments the RT processes involving the interference with the nonresonant tunneling (NRT) channel. The phenomenon has stimulated much experimental [5] and theoretical [6, 7] interest. The coexisting of two possible tunneling channels (resonant and nonresonant), interfering with each other, through the single-electron transistor shows prominent perspectives for the Fano effect [8], i.e., asymmetric line shapes (ALS) in conductance. Because the interference between energetically accessible RT channels and NRT channel has not been investigated. In this sense, it is interesting to ask whether the interference exhibits ALS on satellites peaks and what the characteristic features are manifested in PAT.

On the other hand, the spectral density of current noise, especially its nonequilibrium aspect (so-called shot noise), reveals very useful information hidden in current measurements 9, 10. The study on photon-assisted shot noise (PASN) is developed very fast in recent years. Lesovik and Levitov 11] modelled an open single connected loop threaded by a time-varying Aharonov-Bohm flux to study the flux-controlled interference. This offers a clever way of affecting its dynamics and enables one to study PASN. PAT on nonequilibrium current fluctuation has been observed by Schoelkopf et al. experimentally [12]. To our interest, here, a new degree of freedom is provided by the employment of NRT channel in tunneling processes. The correlation between RT and NRT pro- cesses is high in time for transmitting electrons. The investigation of spectral density of shot noise in PAT has twofold significations. On the one hand, the study of this quantity can be used as a cross-check for the analysis of the interference of the charge transferring, in the case of an existence of continuum level degenerate in energy with a discrete resonant level. On the other hand, the investigation on the zero bias PASN, in which the time average current vanishes, enables us to clarify intrinsic effect of discrete charges in interference with Fano resonance.

In this Letter, we present the theoretical analysis of Fano resonance in PAT and demonstrate those interesting features for the ALS on satellite sidebands. Our analysis is based on a small configuration so called a quantum dot (QD) connected to two electronic reservoirs via tunneling barriers. To capture the essential physics of photon-assisted Fano resonance we consider the simplest case with noninteracting electrons in a single-level QD. The NRT channel is incorporated by a bridge channel as a kind of direct tunneling between the continuum states of two reservoirs, which can be thought as a point-likecontact added to the configuration. This Letter consists of two parts: First, we study the Fano effect in PAT and reveal the characteristics of ALS in peaks located on satellites; Second, we investigate the spectral density of shot noise in photon-assisted Fano resonance.

The system is described by a tunneling Hamiltonian $H=H_{\text {leads }}+H_{d}+H_{l d r}+H_{l r}$, in which $H_{l e a d s}=\sum_{k \alpha} \epsilon_{k \alpha}(t) c_{k \alpha}^{\dagger} c_{k \alpha}, H_{d}=\epsilon_{d}(t) d^{\dagger} d, H_{l d r}=$ $\sum_{k \alpha}\left(t_{k \alpha} c_{k \alpha}^{\dagger} d+t_{k \alpha}^{*} d^{\dagger} c_{k \alpha}\right)$, and $H_{l r}=\sum_{k k^{\prime}}\left(v_{k k^{\prime}} c_{k l}^{\dagger} c_{k^{\prime} r}+\right.$ $\left.v_{k k^{\prime}}^{*} c_{k l}^{\dagger} c_{k^{\prime} r}\right)$, where $c_{k \alpha}^{\dagger}\left(c_{k \alpha}\right)$ creates (annihilates) an electron of momentum $k$ in reservoir $\alpha(=l, r) ; d^{\dagger}(d)$ is the creation (annihilation) operator for an electron in $\mathrm{QD} ; t_{k \alpha}$ are tunneling coefficients that describe the tunneling between the reservoirs $\alpha$ and the quantum dot, while $v_{k k^{\prime}}$ describes the NRT between the reservoirs. The Hamiltonian is time dependent due to energy shifts, $\epsilon_{k \alpha}(t)=\epsilon_{k \alpha}+e V_{\alpha} \cos \omega_{1} t$, of the reservoirs and illuminated QD by irradiation, $\epsilon_{d}(t)=\epsilon_{d}+$ 
$e V_{\omega} \cos \omega_{2} t$. Using formulism of time-dependent Green's functions 13. the current flowed out the left lead is $I_{l}(t)=$ $(2 e / \hbar) \operatorname{Re}\left[\sum_{k k^{\prime}} v_{k k^{\prime}} G_{k r, k^{\prime} l}^{<}(t, t)+\sum_{k} t_{k l} G_{d, k l}^{<}(t, t)\right]$, where $G_{d, k l}^{<}$and $G_{k r, k^{\prime} l}^{<}$are Keldysh Green functions. In the current the term involving $G_{k r, k^{\prime} l}^{<}$contains the NRT and its interference with RT while the term involving $G_{d, k l}^{<}$contains the RT and its interference with NRT. These nonequilibrium Green functions can be evaluated by employing the Keldysh technique and be expressed in terms of Green function $G_{d d}$ at QD and the bare Green functions in the leads. The connection among them in a set of Dyson-like equations includes all possibilities of scattering tunneling and interference. For simplicity, we neglect the energy dependence of various couplings and write the intrinsic linewidth in terms of the density of states, $\rho_{\alpha}$, in the leads $\alpha$ under the wide linewidth approximation, i.e., $\Gamma_{\alpha}=2 \pi \rho_{\alpha}\left|t_{\alpha}\right|^{2}(\alpha=l, r)$ and $W=(2 \pi)^{2} \rho_{l} \rho_{r}|v|^{2}$. Besides, it should be emphasized to point out that there is a mix coupling among the left and right barriers, and the bridge channel over the two reservoirs, i.e., $\Delta=(2 \pi)^{2} \rho_{l} \rho_{r} t_{l} v^{*} t_{r}^{*}=\sqrt{\Gamma_{l} \Gamma_{r} W} e^{i \varphi}$. It is found that the combination of $t_{l} v^{*} t_{r}^{*}$ can be regarded as an existence of intrinsic accesses constructed by RT and NRT channels and gives rise to a phase dependence. The phase $\varphi$ manifests its contribution throughout the following calculations and shows an AharonovBohm-like effect. The time average current is obtained as $I=(e / \pi \hbar) \int d \epsilon \sum_{m n} f_{m n}(\epsilon) T_{m n}(\epsilon)$ with a definition of $f_{m n}(\epsilon)=J_{n}^{2}\left(u_{\omega}\right)\left[f_{l}(\epsilon) J_{m}^{2}\left(u_{l}\right)-f_{r}(\epsilon) J_{m}^{2}\left(u_{r}\right)\right]$, where $J_{n}\left(u_{\beta}\right)$ is the $n$ th-order Bessel function of $u_{\beta}=e V_{\beta} / \hbar \omega_{\beta}$ ( $\beta=\omega, l$ and $r)$ and $f_{\alpha}(\epsilon)$ is the Fermi distribution function of electrons in the reservoir $\alpha . T_{m n}$ describes the transmissions of the tunneling through both RT and NRT channels, the interference between them, and all multiple scattering on the dot and the contacts in the processes of absorption and emission of photons. The analytic formulation is given by

$$
T_{m n}(\epsilon)=\frac{T_{b} \epsilon_{m n}^{2}+\frac{1}{4} \tilde{\Gamma}^{2} \Omega+\epsilon_{m n} \tilde{\Gamma}^{2} \sqrt{T_{b} \Omega} \cos \varphi}{\left(\epsilon_{m n}+\frac{1}{4} \Gamma \sqrt{T_{b} \Omega} \cos \varphi\right)^{2}+\frac{1}{4} \tilde{\Gamma}^{2}}
$$

where $\epsilon_{m n}=\epsilon-\epsilon_{d}+m \omega_{1}-n \omega_{2}, \Gamma=\Gamma_{l}+\Gamma_{r}$, $\tilde{\Gamma}=\Gamma T_{b} / 2\left(1-\sqrt{1-T_{b}}\right)$, and $\Omega^{2}=4 \Gamma_{l} \Gamma_{r} / \Gamma^{2}$. We leave $T_{b}=W /(1+W / 4)^{2}$ as a turnable parameter, socalled transparency of NRT channel, in our following discussions. This is equivalent in controlling the NRT strength. It is a transmission coefficient in the quantum point contact 14]. Ref. 14] has discussed in details that $T_{b}$ varies between zero and one as a function of $W$. In eq.(1) the renormalized levels are given by $E_{m n}=2\left[\epsilon_{d}-m \omega_{1}+n \omega_{2}-\Gamma \sqrt{T_{b} \Omega} \cos \varphi / 4\right] / \tilde{\Gamma}$ and show satellite resonant levels offset from the main nonlinearity by the shifts corresponding to absorption and emission of photons. If we express them in terms of resonant scattering phase shifts $E_{m n}=\cot \delta_{\text {res }}^{m n}$, it is predicted that the sidebands (with the resonant scattering phase shifts in different integer multiples of $\omega_{1}$ and $\omega_{2}$ ) are still remained and but with a shift due to a self energy renormalized the resonance $\Gamma \sqrt{T_{b} \Omega} \cos \varphi / 4$. According to the discussion of Hofstetter et al. []], eq.(1) can be written as a generalized Fano form with an asymmetric parameter, $q=\sqrt{\Omega\left(1-T_{b}\right) / T_{b}} \cos \varphi$ which is independent of PAT. The common Breit-Wigner line shapes of PAT is recovered at $q \rightarrow \infty\left(T_{b}=0\right)$, whereas $q \rightarrow 0\left(T_{b}=1\right)$ yields a symmetric antiresonance. ALS would be explicitly noted at the finite value of $q$. Because of resonant accesses depending on exchange energy of photons, it is predicted that a Fano zero associated with each PAT resonance results in the dips (ALS) on the satellite peaks of current characteristics. Fig.1 displays the current at
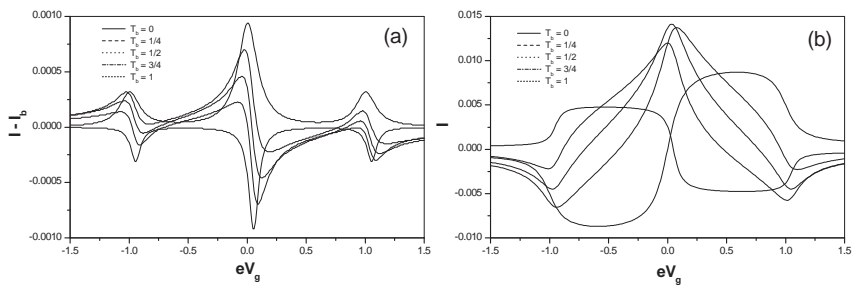

FIG. 1: Current as a function of $e V_{g}$ for $T_{b}=0,0.25,0.5,0.75$, and 1 . The parameters are $u_{\omega}=0, \omega_{1}=1, \varphi=0$, and dc bias $V=0.01$. (a) the symmetric irradiation on the both leads, $u_{l}=u_{r}=1$, and (b) the photon-assisted pumped current under asymmetric illumination, $u_{l}=1$ and $u_{r}=0 . I_{b}$ is the current at $\Gamma_{l}=\Gamma_{r}=0$.

zero temperature as the function of $e V_{g}$, the gate voltage, where the resonant level in QD has been taken as an energy reference, i.e., $\epsilon_{d}=0$. Throughout this Letter, the energy is measured in unit of $\omega_{1}(e=\hbar=1)$ and the symmetric tunneling barriers $\left(\Gamma_{l}=\Gamma_{r}=0.1 \omega_{1}\right)$ are considered. From Fig.1a we find that the satellite peaks are produced at $e V_{g}=n \hbar \omega$ as expected. The amplitude of any given sideband is a non-monotonic function of photon flux in the form of a nontrivial dependence on the Bessel function $J_{n}^{2}\left(u_{\alpha}\right)$ and the interference. Fano line shapes on the sidebands imply that the PAT accesses interfere with the NRT channel. The influence of the Fano resonance has been also represented in Fig.1a by running $T_{b}$ over the regime from the weak NRT to the one that NRT contribution to conductance is comparable in size to the RT component. Increasing $T_{b}$ the current $T_{m n}$ makes the line shape run away from Breit-Wigner line shape $\left(T_{b}=0\right)$ and exhibits a Breit-Wigner dip in the limit when NRT dominates the tunneling $\left(T_{b}=1\right)$. The dips on the line shapes located on satellites saturate at the same finite values symmetrically at the limitation attributed in the NRT channel. It is interesting to notice that the unsynchronized asymmetrizations on the satellite peaks located two sides of main resonance are visible in varying $T_{b}$. Such unsynchronous dips in the absorption and emission of photons can been explained as follows: The scattering cross-section of tunneling is proportional to a function of sum $m$ over $\sin ^{2}\left(\delta_{\text {res }}^{m}+\delta\right)$ []], where $\delta$ is 
a nonresonant phase shift related to the asymmetric parameter $q$, i.e., $q=-\cot \delta$. As pointed above the resonant scattering phase shifts $\delta_{\text {res }}^{m}$ are different for the processes of absorption and emission of photons. Thus the definite value $T_{b}$ ( thus $\delta$ ) results in the different values in $\delta_{\text {res }}^{m}+\delta$ for the accesses of emitting photons (positive $m$ ) and absorbing photons (negative $m$ ). Alternatively, eq.(1) is a sum of two even and one odd functions of $\epsilon$ around its poles. The part of odd function asymmetrizes the line shape and leads to Fano profile. The third term of the numerator in eq. (1) indicates that the processes of absorption and emission of photons affect the Fano profiles in a different weight. It is implicit in the variations of ALS with the strengths of nonresonant transmissions are not synchronous in absorption and emission sidebands. This can be understood as that the tunneling electrons will acquire different coherent phases from the stimulated absorption and emission respectively, which would be exhibited in the interference between RT and NRT channels.

It is well known that an asymmetric illumination in the reservoirs reveals photon-assisted pumping. Without NRT, Kouwenhoven et al. [2] has studied the photonassisted pumping at zero dc bias voltage. We set $V_{r}=0$ and a finite $V_{l}$. The photon absorption occurs only at the left reservoir, which leads to the net current to the right when the RT level above the Fermi level of reservoirs while reverses when RT level below the Fermi level of the reservoirs. Without NRT the pumped current changes sign when the gate voltage is swept such that the RT level moves cross the Fermi level of reservoirs. However, it is not truth when NRT is joined. These processes interfere with NRT shows a wide region of current to the right. The pumped current occurs over a width corresponding to the photon energy. It is noticed that the pumping current is reversed at $T_{b}=1$ with respect to the case of $T_{b}=0$. In Fig.1b we have represented several possibly pumped current profiles with different values of $T_{b}$. Note that except for the limiting situations $T_{b}=0$ and $T_{b}=1$, the profiles are asymmetric. There are two zerocurrent points instead of only one for the case of limiting situations. This behavior originates from an incomplete suppression due to the interference near the degeneracy point of single-particle energies. As one can see in eq. (1), current indicates the Fano line shape is phase dependent and a periodic function of $\varphi$ with period $2 \pi$. The symmetry in $\varphi$ and $-\varphi$ refers to as "phase locking", which is an exact property of two-terminal setups 9 ]. Fig.2 demonstrates the current-phase relation for $T_{b}=0.25$ and 1.00 . From Fig.2a it is evident to note the property that there is a symmetry $I\left(\varphi, e V_{g}\right)$ and $I\left(\varphi-\pi,-e V_{g}\right)$, which follows from the symmetries in electron and hole. It is also interesting to notice that the sidebands are smeared away at $\varphi=\pi / 2$ (see in Fig.2b) because the Fano parameter vanishes at $\varphi=\pi / 2$. It can been seen that we have the usual FAT resonance for $T_{b}=0$ and the disappearance of interference for $T_{b}=1$ at $\varphi=\pi / 2$.
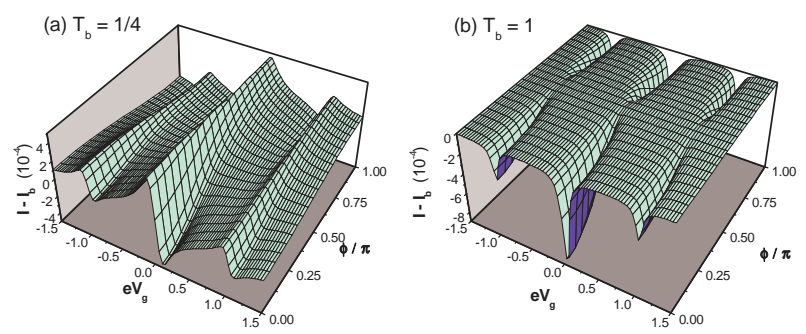

FIG. 2: Current as a function of $e V_{g}$ and $\varphi$ for the symmetric illumination for $T_{b}=$ (a) 0.25 and (b) 1 . Other parameters are the same as those given in Fig.1a

To learn more about the influence offered by the interference with NRT we study the spectral density of shot noise $S(\omega)$ and the effect in affecting its dynamics. It is defined as $S(\omega)=\int d\left(t-t^{\prime}\right) \exp \left[i \omega\left(t-t^{\prime}\right)\right]<$ $\delta \hat{I}(t) \delta \hat{I}\left(t^{\prime}\right)+\delta \hat{I}\left(t^{\prime}\right) \delta \hat{I}(t)>$, where $\delta \hat{I}(t)=\hat{I}(t)-<\hat{I}(t)>$ is the fluctuation in current. In general the noise in PAT oscillates in time with the frequencies of external microwave radiations. $<\delta \hat{I}_{\alpha}(t) \delta \hat{I}_{\beta}\left(t^{\prime}\right)>(\alpha, \beta=l, r)$ does not need to have the same values for different pairs of $\alpha \beta$ in the presence of external irradiation. We calculate $S(\omega)=\sum_{\alpha \beta} S_{\alpha \beta}(\omega)$ instead of $S_{\alpha \beta}(\omega)$. Because of $<\hat{I}_{l}(t)>=-<\hat{I}_{r}(t)>, S(\omega)$ calculated here relates to the zero-point current fluctuation. Using the technique of nonequilibrium Green function as done in calculation of current it makes analytical studies possible. However, the calculation is more complicated because of the correlation among the electrons in the difference processes taking into account. Taking the notions $\tilde{\omega}_{m n}=\omega-\epsilon-\epsilon_{d}+m \omega_{1}-n \omega_{2}$ and $\mathcal{F}_{\beta}\left(\omega, \epsilon, \epsilon^{\prime}\right)=\left(1-f_{\beta}(\epsilon)\right) \delta\left(\omega-\epsilon^{\prime}+\epsilon\right)-f_{\beta}(\epsilon) \delta\left(\omega-\epsilon^{\prime}-\epsilon\right)$, we obtain the spectral density of shot noise, $S(\omega)=$ $\left(e^{2} / \pi \hbar^{2}\right) \sum_{\alpha m n} \int d \epsilon f_{\alpha}(\epsilon) J_{m}^{2}\left(u_{\alpha}\right) J_{n}^{2}\left(u_{d}\right)\left[s_{\alpha}^{m n}(\omega, \epsilon)+\right.$ $\left.\sum_{\beta} \int d \epsilon^{\prime} J_{m}^{2}\left(u_{\beta}\right) J_{n}^{2}\left(u_{d}\right) \mathcal{F}_{\beta}\left(\omega, \epsilon, \epsilon^{\prime}\right) s_{\alpha \beta}^{m n}\left(\omega, \epsilon, \epsilon^{\prime}\right)\right]$, where

$$
\begin{gathered}
s_{\alpha}^{m n}(\omega, \epsilon)=\frac{\Gamma_{\alpha} \tilde{\Gamma}}{\left(\tilde{\omega}_{m n}+\frac{1}{4} \Gamma \sqrt{T_{b} \Omega} \cos \varphi\right)^{2}+\frac{1}{4} \tilde{\Gamma}^{2}} \\
s_{\alpha \beta}^{m n}\left(\omega, \epsilon, \epsilon^{\prime}\right)=\frac{\left[\tilde{\Gamma}_{\alpha}+\frac{1}{4} W \tilde{\Gamma}_{\bar{\alpha}}+\left(-\frac{1}{2}\right)^{\alpha} \Gamma \sqrt{T_{b} \Omega} \sin \varphi\right] \tilde{\Gamma}_{\beta}}{\left(\tilde{\epsilon}_{m n}+\frac{1}{4} \Gamma \sqrt{T_{b} \Omega} \cos \varphi\right)^{2}+\frac{1}{4} \tilde{\Gamma}^{2}}(3)
\end{gathered}
$$

The dc and zero-frequency spectral density of shot noise at a finite dc bias and zero temperature as the function of gate voltage is plotted in Fig.3. It contains a new information as compared with the current in the evolution of the transparency of NRT, $T_{b}$. By analyzing the asymmetric variation of shapes with varying the transparency, $T_{b}$, it is found that the spectral density of shot noise recovers its original form at $T_{b}=1$ as that at $T_{b}=0$ and reverses shape at $T_{b}=0.5$. The result is different from what has been seen in the current. The phase-dependence has been illustrated in Fig.4. This is a 


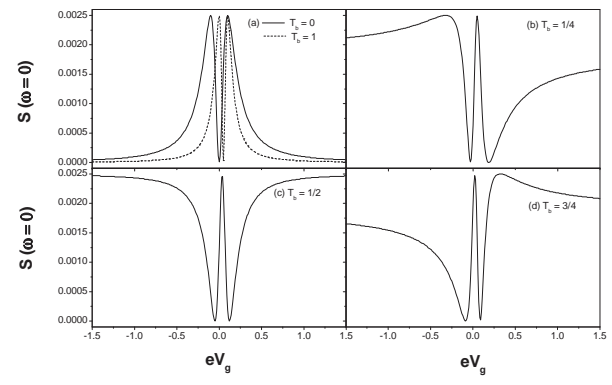

FIG. 3: Spectral density of shot noise as a function of $e V_{g}$ in absence of irradiation $\left(u_{l}=u_{r}=u_{\omega}=0\right)$ for $T_{b}=0,0.25$, $0.5,0.75$, and $1 ; \varphi=0$ and dc bias $V=0.01$.
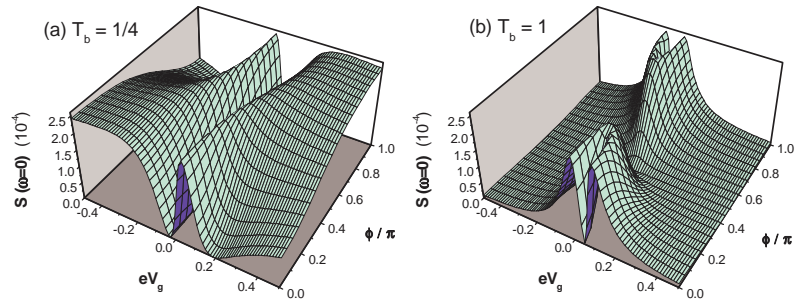

FIG. 4: Spectral density of shot noise as a function of $e V_{g}$ and $\varphi$ for the case in Fig. 3 at $T_{b}=$ (a) 0.25 and (b) 1 .

robust feature which survives for all Fano resonant tunneling in a structure of QD. It is an unambiguous signature of the fact of Aharonov-Bohm-like oscillation. The difference between here and the description of Lesovik and Levitov 11 is that the phase dependence is not an external flux but the intrinsic existence. The shot noise remains the constant at $\varphi=\pi / 2$ and $T_{b}=1$ in Fig. $4 \mathrm{~b}$ indicates the interference smearing over fluctuation and leaving noise as a constant background. This is agreement with that we have observed in the current in Fig.2b. The fact reveals the symmetry of phase dependence is the intrinsic property and is independent of the absorption and emission of photons.

Before making adieu we would like to avail ourselves of a little space to face an interesting question: what the photon-assisted signature in the shot noise may be and what effect does nonresonant channel give risen in? To this we plot the spectral density of shot noise at zero bias voltage in Fig.5. Because no time-average current exists when the ac voltage drop is the same for both reservoirs [2] the fluctuations on the current are completely induced by the external radiations. From the plot of shot noise in Fig.5, the predicted modulation respect to the absorbtion and emission of photons does arise. It is seen that a continuing increase of transparency of NRT channel, the varying on the top of peaks in the zero-bias spectral density of shot noise does also reveal the asymmetric Fano resonance. It indicates the possibilities of relaxation among these states excited by absorbtion and emission of photons. An electron absorbs a photon or emits a photon and hops to a energetically accessible above the Fermi level in the leads. The interference with the forward and backward possibility in the nonresonant channel leads to the ALS in the zero-bias shot noise. The phase dependence of zero-bias shot noise also shows the fact that the interference smears over fluctuation and leaves noise as a constant background.

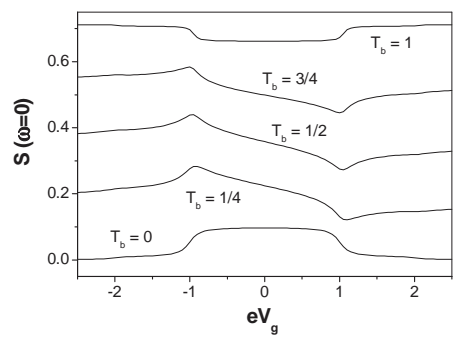

FIG. 5: Spectral density of shot noise as a function of $e V_{g}$ in present of irradiation for $T_{b}=0,0.25,0.5,0.75$, and 1 at zero bias voltage. The parameters are $\varphi=0, u_{\omega}=0$, and $u_{l}=u_{r}=1$.

In summary, we have demonstrated that the shape of Fano profile appears in peaks located on absorption and emission sidebands. The asymmetrization of peaks on those satellites, corresponding to absorbing and emitting photons respectively, are not synchronous in varying the strengths of nonresonant transmissions. Both the current and spectral density of shot noise depend on a phase which is essentially a sore of intrinsic phase difference between the energetically accessible RT and NRT channels.

We acknowledge the support from NNSFC and NSF of Guangdong Province under Grant No. 90103027, 10274069 , and 011151.

[1] L.P. Kouwenhoven et al., Phy. Rev. B50, 2019(1994); Phy. Rev. Lett. bf 73, 3443(1994); T.H. Oosterkamp, et al., ibid, 78, 1536(1997); R.H. Blik et al., Appl. Phys. Lett. 67, 3924(1995).

[2] P.L. McEuen et al., Nanotechnology 7, 406(1996).

[3] J. Göres et al., Phys. Rev. B62, 2188(2000).

[4] I.G. Zacharia et al., Phys. Rev. B64, 155311(2001).

[5] V. Madhavan et al., Science 280, 567(1998); H.C. Manoharan et al., Nature (London) 403, 512(2000); J. Li, et al., Phys. Rev. Lett. 80, 2893(1998); N. Knorr, et al., ibid, 88, 96804(2002).

[6] E. Tekman, et al., Phys. Rev. B48, 2553(1993); J.U. Nöckel, et al., ibid, B53, 17415(1994).

[7] O.Újsághy, et al., Phys. Rev. Lett. 85, 2557(1998); B.R. Bulka, et al., ibid, 86, 5128(2001); W. Hofstetter, et al., ibid, 87, 156803(2001).

[8] U. Fano, Phys. Rev. 124, B1866(1961).

[9] M. Büttiker, Phys. Rev. Lett. 65, 2901(1990).

[10] G. Iannaccone et al., Phys. Rev. Let. 80, 1054(1998); T. Gramespacher and M. Büttiker, ibid, 81, 2763(1998).

[11] G.B. Lesovik and L.S. Levitov, Phys. Rev. Lett. 72, 538(1994).

[12] R.J. Schoelkopf et al., Phys. Rev. Lett. 80, 2437(1998).

[13] A.P. Jauho, et al., Phys. Rev. B50, 5528 (1994). 
[14] J.C. Cuevas, et al., Phys. Rev. B54, 7366(1996). 